EPJ Web of Conferences 67, 02125 (2014)

DOI: $10.1051 /$ epjconf / 20146702125

(c) Owned by the authors, published by EDP Sciences, 2014

\title{
Collision of a small bubble with a large falling particle
}

\author{
Jiri Vejrazka ${ }^{1, a}$, Martin Hubicka ${ }^{2}$, and Pavlina Basarova ${ }^{2}$ \\ 1 Institute of Chemical Process Fundamentals, 16502 Praha 6, Czech Republic \\ 2 Prague Institute of Chemical Technology, Department of Chemical Engineering, 16628 Praha 6, Czech Republic.
}

\begin{abstract}
The motion of a tiny bubble $(<1 \mathrm{~mm})$ in a neighborhood of a solid sphere falling through a liquid is studied. A model assuming irrotational flow around the sphere and spherical bubble shape is provided; this model is validated by comparison with the experiment. The model can be further simplified by neglecting inertial forces, which are negligible in present experiments. Results of the model are provided also for the opposite limit, in which the inertial forces are dominating the bubble motion.
\end{abstract}

\section{Introduction}

Motivated by applications of separation of various plastic materials by flotation during their recycling, we study the behavior of a tiny $\left(D_{e}<1 \mathrm{~mm}\right)$ bubble rising in water, which encounters a larger spherical particle falling through the liquid (with velocity $U_{p}$ in order of few $\mathrm{cm} / \mathrm{s}$ ). The aim is to determine, what are the conditions under which the bubble touches the particle's surface. Basically, all bubbles, which are initially in a distance $x_{0}$ (see figure 1) smaller than some limiting value $x_{g}$ collide with the particle surface, whereas bubbles with $x_{0}>x_{q}$ do not collide. The aim hence reduces in an effort of obtaining an expression for the maximum collision distance $x_{g}$.

In paper by Hubicka et al.[1], we have experimentally investigated the collision. We have provided a model for the bubble motion, which was based on expressing hydrodynamic forces acting on the bubble. We have also shown that for studied conditions of small particle velocity (which

\footnotetext{
a e-mail: vejrazka@icpf.cas.cz
}

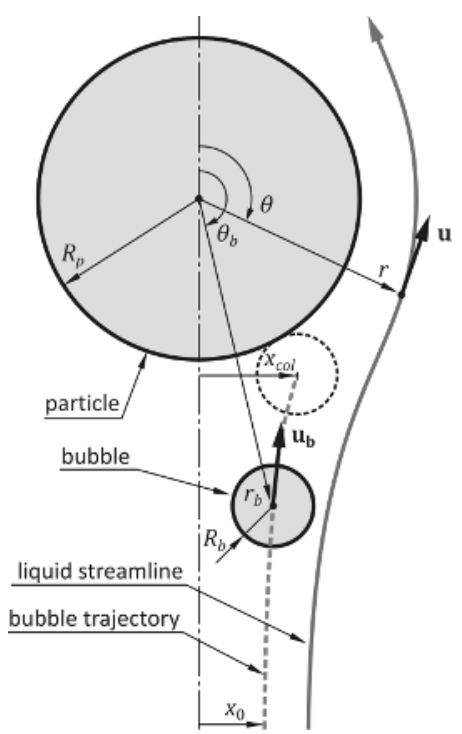

Fig. 1. Studied configuration and symbols. are typical for practical conditions), the model can be further simplified by considering only the drag and buoyancy forces. In this conference contribution, we will summarize our previous work [1]. In addition, we will show results of the model for the hypothetical opposite case, in which the bubble motion is dominated only by inertial forces, and the drag and buoyancy can be neglected.

\section{Experimental observations}

In experiments, a single bubble ( 0.5 to $0.8 \mathrm{~mm}$ in diameter) was created in a controlled manner [2] in a glass cell (height $50 \mathrm{~cm}$, width $8 \mathrm{~cm}$ and depth $6 \mathrm{~cm}$ ). A glass ball (14.1 $\mathrm{mm}$ in diameter) was fixed to a traversing device, which displaced it downward with a velocity of 5 or $10 \mathrm{~cm} / \mathrm{s}$. A high-speed camera (Redlake Motion Pro) was moved by another traversing device with the same velocity as the glass particle. Movies of the bubble motion around the particle (recorded with a rate of 500 frames per second, resolution $1280 \times 1024$ pixels and scale $14 \mu \mathrm{m}$ ) were analyzed using NIS-Elements software; this analysis provided data about the bubble size and its trajectory. Experiments were repeated with several bubble sizes, two particle velocities and two different working fluids. For each operating condition, several trajectories differing in the initial bubble position $x_{0}$ were recorded, leading to a set of experimental trajectories, whose example is shown in figure 2. More details about experiments are provided in [1].

A remarkable behavior observed in experiments is the linear dependence of the bubble position at the instant of collision with the particle surface, on the initial position, $x_{c o l}=k x_{0}$, as it is illustrated in figure 3 .

\section{Model}

In this contribution, we consider three different models for the bubble motion. The full model solves the bubble trajectory in the frame of reference, which moves with the particle (figure 1). This model is simplified by omitting some terms, leading to either non-inertial or inviscid models. 

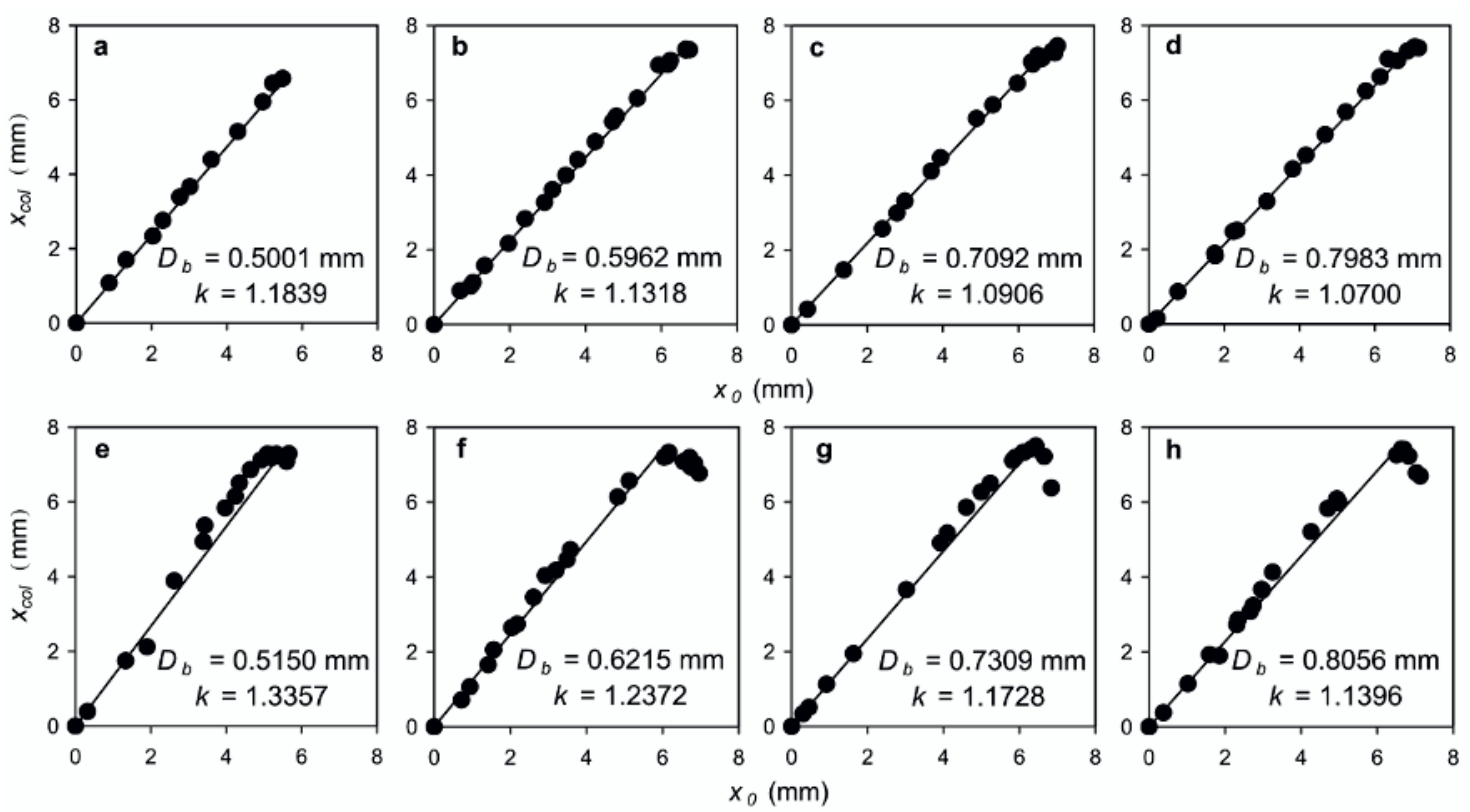

Fig. 3. Observed dependence of the radial distance $x_{c o l}$ at the time of collision with the particle, on the initial position $x_{0}$ of the bubble. Data for pure water, particle velocity $50 \mathrm{~mm} / \mathrm{s} \mathrm{(a-d)} \mathrm{and} 100 \mathrm{~mm} / \mathrm{s}$ (e-h).

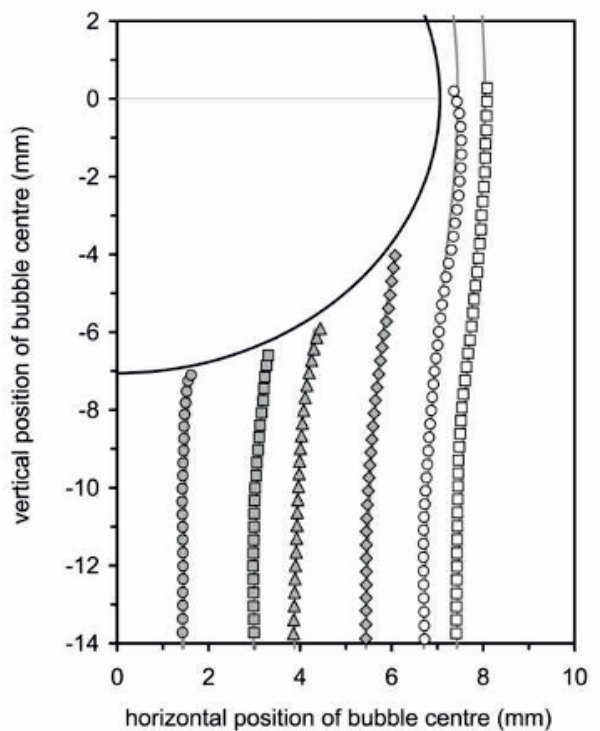

Fig. 2. Example of observed and computed trajectories of bubble. Velocity of the ball is $50 \mathrm{~mm} / \mathrm{s}$, bubble diameter $0.596 \mathrm{~mm}$, pure water.

\subsection{Full model}

The full model assumes that the liquid motion around the sphere is not affected by the bubble, and is modeled by an irrotational flow. Such an assumption is reasonable if the Reynolds number of the sphere is high enough,

$$
R e_{p}=2 \frac{\rho U_{p} R_{p}}{\mu} \gg 1,
$$

and values $R e>100$ are generally sufficient ( $\rho$ and $\mu$ are the liquid density and viscosity). It is also assumed that the impulse related with the bubble motion is smaller than the impulse related with the motion of the sphere $\left(\rho R_{b}^{3} U_{b} \ll\right.$ $\left.\rho R_{p}^{3} U_{p}\right)$. The assumption of irrotational flow holds reasonably only upstream the sphere (i.e. in its lower part), but is not justified downstream the sphere, where the flow separates. Another assumption is that the bubble remains spherical. In low-viscosity fluids, this assumption is satisfied if the Weber number of the bubble remains small during its motion,

$$
W e=2 \frac{\rho R_{b}\left|\mathbf{u}_{\mathbf{b}}-\mathbf{u}\right|^{2}}{\sigma} \ll 1,
$$

where $\sigma$ is the surface tension. This assumptions is the first to be unsatisfied in the present works. On other hand, the present paper is valid also for motion of some light spherical particles around a larger moving particle; the assumption about the Weber umber is not required in such a case.

The bubble trajectory is obtained by solving an equation for bubble motion. This equation is written as a balance of forces, which act on the bubble. Those forces include the buoyancy, drag, added-mass, inertia and lift,

$$
\mathbf{F}_{\mathrm{b}}+\mathbf{F}_{\mathrm{d}}+\mathbf{F}_{\mathrm{am}}+\mathbf{F}_{\mathrm{I}}+\mathbf{F}_{\mathbf{L}}=\mathbf{0}
$$

The added-mass force $\mathbf{F}_{\mathbf{a m}}$ is proportional to the bubble acceleration, while other forces are either constant or dependent on the bubble velocity. The balance (3) is hence a differential equation of second order (for each position component). Detailed expressions of considered forces are provided in [1].

The force balance (3) is not complete, as some forces (e.g. the history force [3]) are neglected. The model compares well with the experimental data, however. This is apparent from figure 2, in which experimental trajectories (points) are drawn above results of the full model (lines, mostly hidden below the symbols). Furthermore, the velocity evolution of the bubble is also captured correctly, as it is apparent from figure 4, where modeled and experimental velocity of the bubble are compared.

Figure 5 shows the computed trajectories of the full model in the left part of the images by dark lines. At very high velocities of the particle (e.g. figure $5 \mathrm{~d} 1$ and partly also figure $5 \mathrm{~d} 2$ ), the full model predicts that the bubble is expelled by the particle in the way that it does not come in 
contact with the particle surface. This is mostly due to the inertial force $\mathbf{F}_{\mathbf{I}}$ in front of the bubble.

It is noted that a similar model for motion of small particles around larger bubbles was presented recently by Huang et al. [6].

\subsection{Non-inertial model}

A remarkable behavior [1] observed in experiments (and in results of the computations by the full model for low particle velocities) is the linear dependence of the radial bubble position at the instant of collision with the particle surface, on the initial radial position, $x_{c o l}=k x_{0}$. This simple dependence is easily explained if we consider for the bubble velocity $\mathbf{u}_{\mathbf{b}}$ at each instant

$$
\mathbf{u}_{\mathbf{b}}=\mathbf{u}+\mathbf{u}_{\mathbf{b}, \mathbf{0}},
$$

where $\mathbf{u}$ is the local liquid velocity in the location of the bubble and $\mathbf{u}_{\mathbf{b}, \mathbf{0}}$ is the velocity of bubble rise in a still liquid. Equation (4) follows from (3) if only buoyancy $\mathbf{F}_{\mathbf{b}}$ and $\operatorname{drag} \mathbf{F}_{\mathbf{d}}$ are considered, whereas $\mathbf{F}_{\mathbf{a m}}, \mathbf{F}_{\mathbf{I}}$ and $\mathbf{F}_{\mathbf{L}}$ are neglected. The trajectory of the bubble is then easily derived via the stream function. The stream function $\psi$ of fluid particles moving around the sphere is given by

$$
\psi=\frac{1}{2}\left(1-\frac{R_{p}^{3}}{r^{3}}\right) U_{p} r^{2} \sin ^{2} \theta .
$$

The bubble trajectory function $\psi_{b}$ is computed. This function is constant along the bubble trajectory, and is hence analogous to stream function $\psi$, which is constant along the trajectory of the liquid. It is expressed by adding the term $\frac{1}{2} U_{b, 0} r^{2} \sin ^{2} \theta$ to $\psi$; this term corresponds to the motion with constant velocity $U_{b, 0}$ aligned with the particle axis. We hence obtain

$$
\psi_{b}=\frac{1}{2}\left(U_{p}+U_{b, 0}\right)\left[1-\frac{R_{p}^{3}}{\left(1+U_{b, 0} / U_{p}\right) r_{b}^{3}}\right] r_{b}^{2} \sin ^{2} \theta
$$

Note that $\psi_{b}$ has the same form as the stream function $\psi$ of the flow around a sphere, but the velocity is different $\left(U_{p}+\right.$ $\left.U_{b}\right)$, as well as the sphere radius $\left(R_{p}\left(1+U_{b, 0} / U_{p}\right)^{-1 / 3}\right)$.

The collision occurs when the bubble distance from the particle center is $r_{b}=R_{p}+R_{p}$. Using (6), we can now easily derive an expression for the collision position $x_{c o l}$, which is

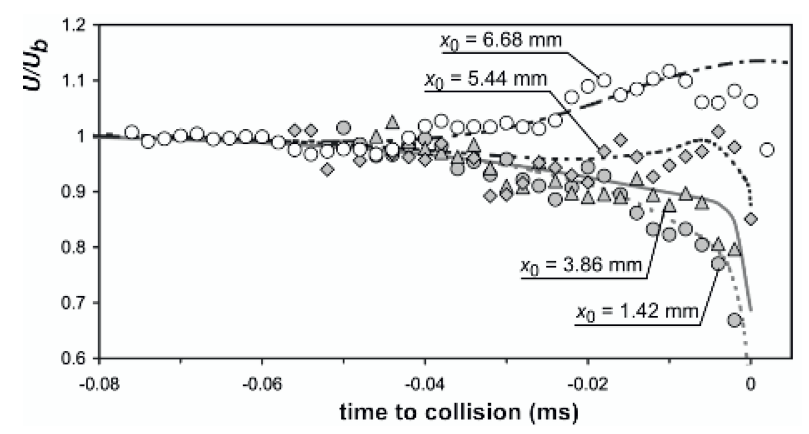

Fig. 4. Comparison of the bubble velocity computed using the full model and experimental data. found (in agreement with experiments) in the form $x_{c o l}=$ $k x_{0}$, where the constant $k$ is

$$
k=\left[1-\frac{R_{p}^{3}}{\left(1+U_{b, 0} / U_{p}\right)\left(R_{p}+R_{b}\right)^{3}}\right]^{-\frac{1}{2}}
$$

The maximum initial distance $x_{g}$, for which the bubble just touches the particle surface, is easily determined as

$$
x_{g}=\frac{R_{p}+R_{b}}{k} \text {. }
$$

The presented non-inertial model is basically a combination of the classical models of Sutherland [4] and of Flint and Howarth [5], applied to the case of moving bubbles instead of settling particles, for which those models have been developed. Its results are also shown in Figure 5 (gray lines in the left parts of the trajectory maps, which are frequently overlapped by black lines). For the results seen in the second row of figure 5 (results for water), the non-inertial model predicts correctly the bubble trajectory for velocities up to $10 \mathrm{~cm} / \mathrm{s}$, which is the case of experiments [1].

\subsection{Inviscid model}

The remaining terms of the motion equation (3) are studied by neglecting the buoyancy and drag forces, $\mathbf{F}_{\mathbf{b}}$ and $\mathbf{F}_{\mathbf{d}}$. All remaining forces $\left(\mathbf{F}_{\mathbf{a m}}, \mathbf{F}_{\mathbf{I}}\right.$ and $\left.\mathbf{F}_{\mathbf{L}}\right)$ follow from the inertia of the liquid, and such a model is hence purely inertial. The rising motion of the bubble is now assured by imposing the velocity $\mathbf{U}_{\mathbf{b}, 0}$ via initial conditions; because the drag and buoyancy are neglected, the initial velocity is maintained up to the interaction with the particle.

The resulting trajectories are shown in figure 5 in the right halves of the trajectory maps. For small particle velocity, the bubbles continue due to its inertia (represented by the added-mass force $\mathbf{F}_{\mathbf{a m}}$ ) straight ahead and hits the particle with unchanged velocity (figure 5a). At higher velocities $\mathbf{U}_{\mathbf{p}}$, the bubble trajectory is deflected due to the inertial force $\mathbf{F}_{\mathbf{I}}$ acting on the bubble (figures $5 \mathrm{~b} 2$ and $5 \mathrm{c} 2$ ). This inertial force prevents the bubbles to come close to the particle surface. As the particle velocity is increased, the distance, in/to which the bubbles are deflected, is increasing (compare figures 5b2 and 5c2); however, at high velocities, this distance is then increasing only very slowly (figures $5 \mathrm{c} 2$ and $5 \mathrm{e} 2$ ) in consequence of the character of the inertial force $\mathbf{F}_{\mathbf{I}}$, which decreases with the distance from the particle as $\sim r^{-4}$.

The inertial model shows another feature, which might be easily overlooked in figure 5: close the particle's equator $\left(\theta=90^{\circ}\right)$, the inertial force $\mathbf{F}_{\mathbf{I}}$ attracts the bubbles toward the particle. This is seen from the curvature of trajectories of the bubble, which bend toward the particle's surface close to the particle's equator.

Note that the inviscid approach is useful only for understanding the effect of inertial forces. This limiting case is applicable directly only under exotic conditions (either very low liquid viscosity, or high particle velocity combined with very high surface tension). 
a1) $U_{p}=0.0093 \mathrm{~m} / \mathrm{s}, \mu=9.1 \cdot 10^{-5} \mathrm{~Pa} . \mathrm{s}$

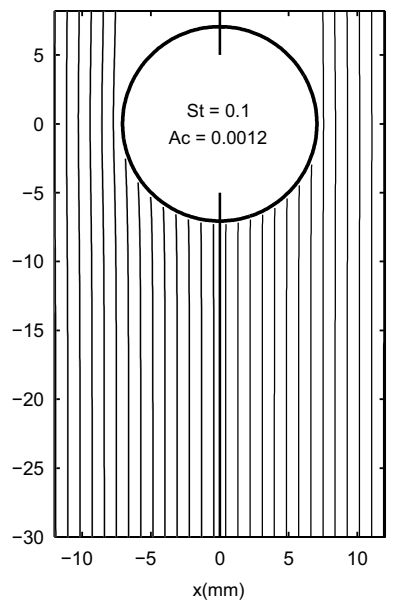

a2) $U_{p}=0.0093 \mathrm{~m} / \mathrm{s}, \mu=9.1 \cdot 10^{-4} \mathrm{~Pa} . \mathrm{s}$

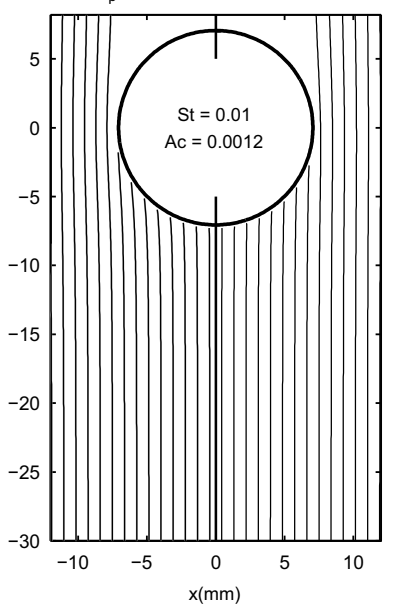

a3) $U_{p}=0.0093 \mathrm{~m} / \mathrm{s}, \mu=9.1 \cdot 10^{-3} \mathrm{~Pa} . \mathrm{s}$

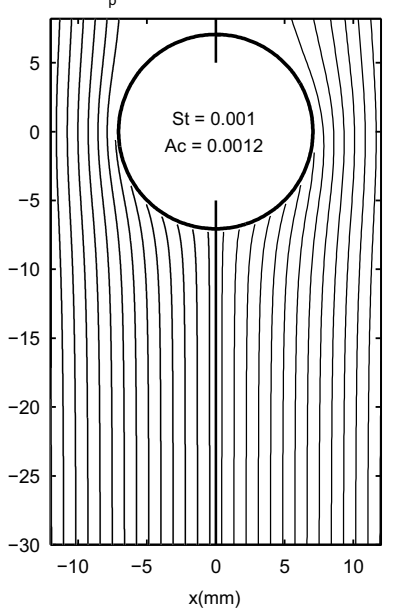

b1) $U_{p}=0.093 \mathrm{~m} / \mathrm{s}, \mu=9.1 \cdot 10^{-5} \mathrm{~Pa} . \mathrm{s}$

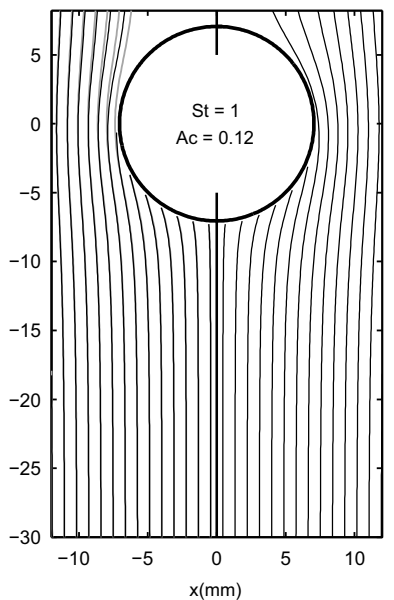

b2) $U_{p}=0.093 \mathrm{~m} / \mathrm{s}, \mu=9.1 \cdot 10^{-4} \mathrm{~Pa} . \mathrm{s}$

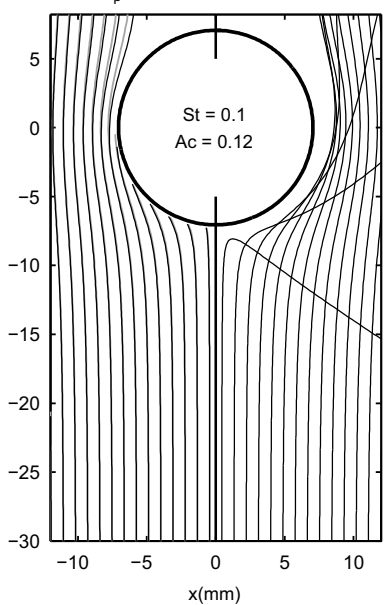

b3) $U_{p}=0.093 \mathrm{~m} / \mathrm{s}, \mu=9.1 \cdot 10^{-3} \mathrm{~Pa} . \mathrm{s}$

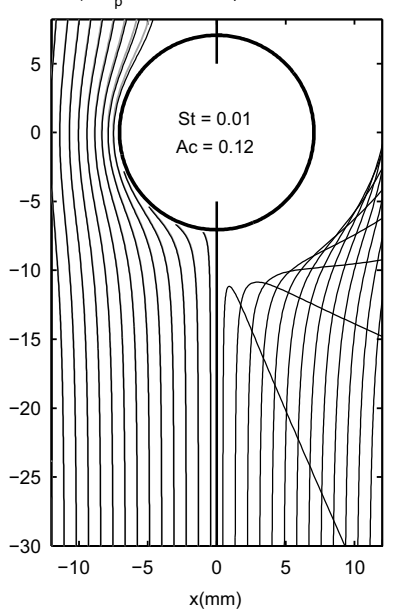

c1) $U_{p}=0.93 \mathrm{~m} / \mathrm{s}, \mu=9.1 \cdot 10^{-5} \mathrm{~Pa} . \mathrm{s}$

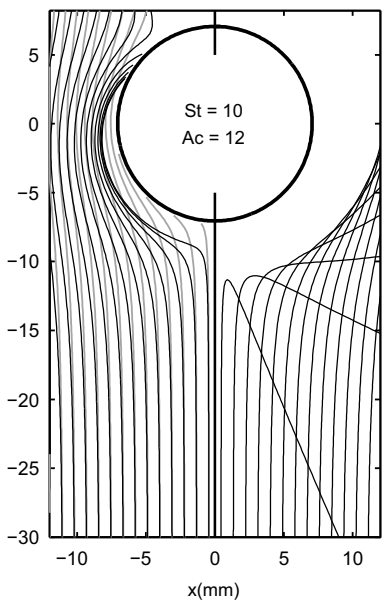

c2) $U_{p}=0.93 \mathrm{~m} / \mathrm{s}, \mu=9.1 \cdot 10^{-4} \mathrm{~Pa} . \mathrm{s}$

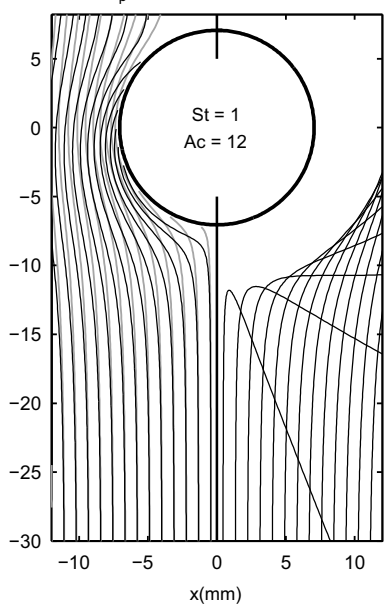

c3) $U_{p}=0.93 \mathrm{~m} / \mathrm{s}, \mu=9.1 \cdot 10^{-3} \mathrm{~Pa} . \mathrm{s}$

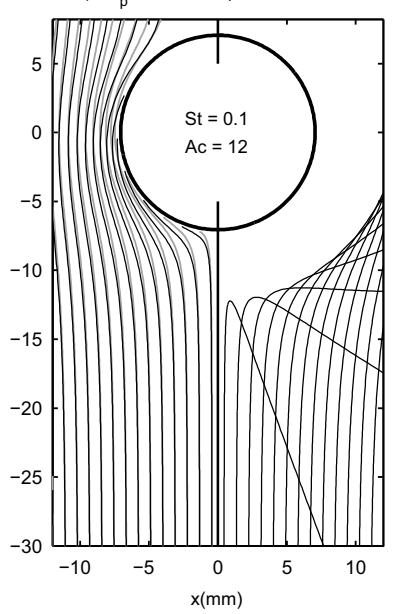

d1) $U_{p}=9.3 \mathrm{~m} / \mathrm{s}, \mu=9.1 \cdot 10^{-5} \mathrm{~Pa} . \mathrm{s}$

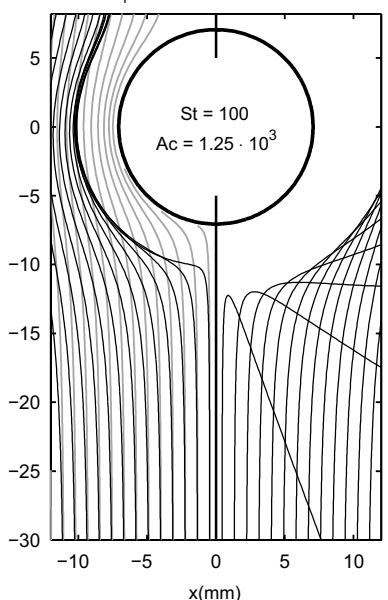

d2) $U_{p}=9.3 \mathrm{~m} / \mathrm{s}, \mu=9.1 \cdot 10^{-4} \mathrm{~Pa} . \mathrm{s}$

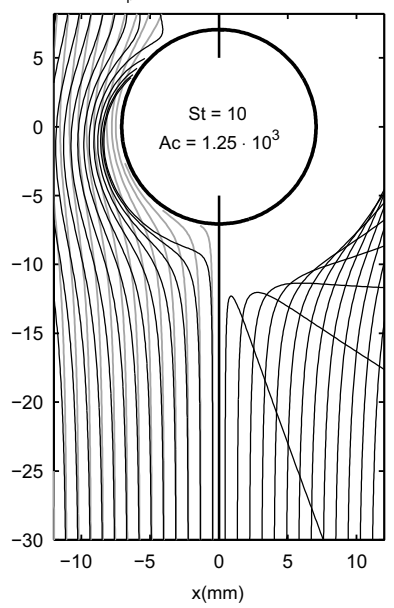

d3) $U_{p}=9.3 \mathrm{~m} / \mathrm{s}, \mu=9.1 \cdot 10^{-3}$ Pa.s

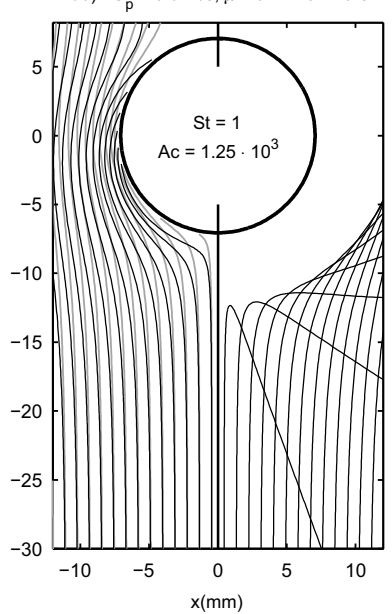

Fig. 5. Computed trajectories of $0.5 \mathrm{~mm}$ bubble, encountering $14.1 \mathrm{~mm}$ particle moving at velocity $U_{p}$ Each row is for a different liquid viscosity $(0.091,0.91$ and $9.1 \mathrm{mPa} \cdot \mathrm{s}$ for the first, second and third row, respectively; the first row is a rather hypothetical case). All results are for a bubble with immobile interface (e.g. contaminated with surfactants). Left halves of the trajectory map shows the results for the full model (black lines) and for the non-inertial model (gray lines, which sometimes hidden below the black lines). The right halves show the results of the inviscid model. 


\subsection{Usability of simplified models}

The non-inertial model is easy to use. It can be used under two conditions: i) the inertial force $\mathbf{F}_{\mathbf{I}}$ (which is responsible from deflecting the bubble from the particle) is negligible compared to the buoyancy (which drives the bubble upward), and ii) the bubble, when perturbed from its motion with a velocity of steady rise $U_{b, 0}$ relative to the liquid, relaxes rapidly back to this equilibrium velocity.

The first condition is readily expressed by comparing the two forces. The inertial force is proportional to $\rho R_{b}^{3} a$, where $a$ is the liquid (convective) acceleration, which scales as $\sim U_{p}^{2} / R_{p}$. The ratio of the inertial and buoyancy forces is hence proportional to

$$
A c=\frac{a}{g}=\frac{U_{p}^{2}}{g R_{p}} .
$$

If this dimensionless parameter is small, $A c \ll 1$, bubble can follow the motion predicted by the non-inertial model. Oppositely, if $A c \gg 1$, the bubble will be deflected by the inertial force $\mathbf{F}_{\mathbf{I}}$ (see e.g. figure $5 \mathrm{c} 3$, where the trajectories of the full and non-inertial model deviate).

The second condition is well-known and it is commonly expressed by means of Stokes number,

$$
S t=\frac{1}{9} \frac{\rho U_{p} R_{b}^{2}}{\mu R_{p}}
$$

which is the ratio of the relaxation time $\left(\sim \rho R_{b}^{2} /(9 \mu)\right)$ and time scale of action of perturbating forces $\left(\sim R_{p} / U_{p}\right)$. As it is apparent from figure 5, the non-inertial model holds only if $S t \ll 1$. Oppositely, for very high $S t$, the bubble trajectory approaches those predicted by the inviscid model.

The inviscid model itself would be valid only under very exotic conditions. Still, it is interesting to observe its results, as their detailed examination allows us to understand the differences of the full and non-inertial models, and eventually provide a simplified equivalent of the full model.

\section{Conclusions}

We have provided three different models for the motion of a spherical bubble around a large moving particle. The full models agree well with the experiments. In most practical cases, much simpler non-inertial model can provide reliable and accurate results; for its validity, both the acceleration and Stokes numbers should be small. The nonviscous model predicts that bubbles do not come in contact with the particle, if its velocity is high. This model is valid, however, only in rather exotic situations.

\section{Acknowledgement}

This research was supported by Czech Science Foundation (P101/11/0806).

\section{References}

1. M. Hubicka, P. Basarova J. Vejrazka, Int. J. Mineral Proc. 121, 21 (2013)

2. J. Vejrazka, M. Fujasova, P. Stanovsky, M. Ruzicka, J. Drahos, Fluid Dyn. Res. 40, 521 (2008)

3. J. Magnaudet, I. Eames, Annu. Rev. Fluid Mech. 32, 659 (2000)

4. K. L. Sutherland, J. Phys. Chem. 52, 394 (1948)

5. L. R. Flint, W. J. Howarth, Chem. Eng. Sci. 26, 1155 (1971)

6. Z. Huang, D. Legendre, P. Guiraud, Chem. Eng. Sci. 68, 1 (2012) 